\title{
Buprenorphine maintenance versus placebo or methadone maintenance for opioid dependence (Review)
}

\author{
Mattick RP, Kimber J, Breen C, Davoli M
}

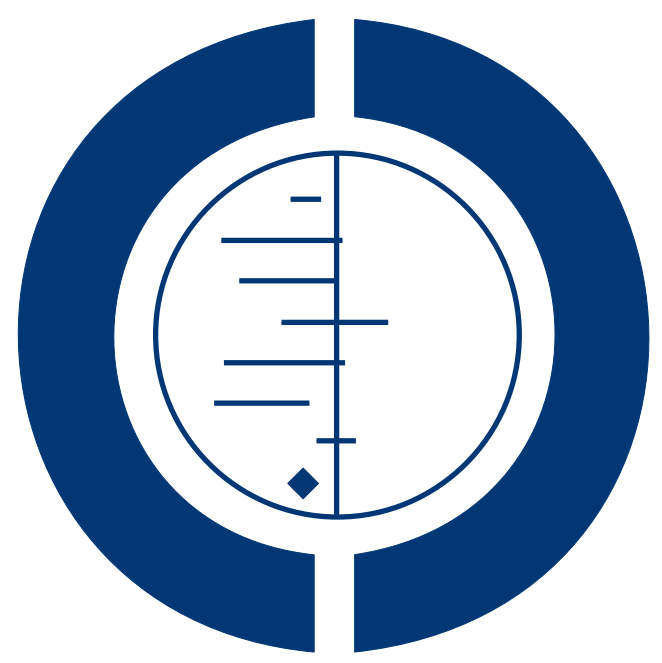

\section{THE COCHRANE COLLABORATION $^{\circledR}$}

This is a reprint of a Cochrane review, prepared and maintained by The Cochrane Collaboration and published in The Cochrane Library 2003, Issue 2

http://www.thecochranelibrary.com

\section{WILEY}

Buprenorphine maintenance versus placebo or methadone maintenance for opioid dependence (Review)

Copyright $\odot 2008$ The Cochrane Collaboration. Published by John Wiley \& Sons, Ltd. 
TABLE OF CONTENTS

HEADER . . . . . . . . . . . . . . . . . . . . . . . . . . . . . . . . . . . . . . . . 1

ABSTRACT . . . . . . . . . . . . . . . . . . . . . . . . . . . . . . . . . . . . . . . . . . . . . . .

PLAIN LANGUAGE SUMMARY . . . . . . . . . . . . . . . . . . . . . . . . . . . . . . . . . . . 2

BACKGROUND . . . . . . . . . . . . . . . . . . . . . . . . . . . . . . . . . . . . . .

OBJECTIVES . . . . . . . . . . . . . . . . . . . . . . . . . . . . . . . . . . . . . .

RESULTS . . . . . . . . . . . . . . . . . . . . . . . . . . . . . . . . . . . . . . .

DISCUSSION . . . . . . . . . . . . . . . . . . . . . . . . . . . . . . . . . . . . . 5

AUTHORS' CONCLUSIONS . . . . . . . . . . . . . . . . . . . . . . . . . . . . . . . 5

ACKNOWLEDGEMENTS . . . . . . . . . . . . . . . . . . . . . . . . . . . . . . . . . . . . . . 6

REFERENCES . . . . . . . . . . . . . . . . . . . . . . . . . . . . . . . . . . . . . . 6

SOURCES OF SUPPORT . . . . . . . . . . . . . . . . . . . . . . . . . . . . . . . . . . . . . . . .

INDEX TERMS . . . . . . . . . . . . . . . . . . . . . . . . . . . . . . . . . . . . 8

Buprenorphine maintenance versus placebo or methadone maintenance for opioid dependence (Review)

Copyright $\odot 2008$ The Cochrane Collaboration. Published by John Wiley \& Sons, Ltd. 


\title{
[Intervention Review] \\ Buprenorphine maintenance versus placebo or methadone maintenance for opioid dependence
}

\author{
RP Mattick, J Kimber, C Breen, M Davoli \\ Contact address: A/Prof. Richard P. Mattick, Director of Research, National Drug and Alcohol Research Centre, University of New South \\ Wales, National Drug and Alcohol Research Centre, University of New South Wales, Sydney, New South Wales, 2052, AUSTRALIA. \\ R.Mattick@unsw.edu.au.
}

Editorial group: Cochrane Drugs and Alcohol Group.

Publication status and date: Unchanged, published in Issue 1, 2008.

Review content assessed as up-to-date: .

Citation: Mattick RP, Kimber J, Breen C, Davoli M. Buprenorphine maintenance versus placebo or methadone maintenance for opioid dependence. Cochrane Database of Systematic Reviews 2003, Issue 2. Art. No.: CD002207. DOI: 10.1002/14651858.CD002207.pub2.

Copyright (C) 2008 The Cochrane Collaboration. Published by John Wiley \& Sons, Ltd.

\begin{abstract}
A B S T R A C T
Background

Buprenorphine has been reported as an alternative to methadone and LAAM for maintenance treatment of opioid dependence, differing results are reported concerning its relative effectiveness indicating the need for an integrative review.
\end{abstract}

\section{Objectives}

To evaluate the effects of buprenorphine maintenance against placebo and methadone maintenance in retaining patients in treatment and in suppressing illicit drug use.

\section{Search strategy}

We searched the following databases up to 2001, inclusive: Cochrane Drugs and Alcohol Review Group Register, the Cochrane Controlled Trials Register, MEDLINE, EMBASE, Current Contents, Psychlit, CORK [www. state.vt.su/adap/cork], Alcohol and Drug Council of Australia (ADCA) [www.adca.org.au], Australian Drug Foundation (ADF -VIC) [www.adf.org.au], Centre for Education and Information on Drugs and Alcohol (CEIDA) [www.ceida.net.au], Australian Bibliographic Network (ABN), Library of Congress databases, available NIDA monographs, the College on Problems of Drug Dependence Inc. proceedings, the reference lists of all identified studies and published reviews. Authors of identified RCT's were asked about any other published or unpublished relevant RCT.

\section{Selection criteria}

Randomised clinical trials of buprenorphine maintenance versus either placebo or methadone maintenance for opioid dependence.

\section{Data collection and analysis}

Reviewers evaluated the papers separately and independently, rating methodological quality of concealment of allocation; data were extracted independently for meta-analysis and double-entered.

\section{Main results}

Thirteen studies met the inclusion criteria, all were randomised clinical trials, all but one were double-blind. The method of concealment of allocation was not clearly described in 11 of the studies, otherwise methodological quality was good. Buprenorphine given in flexible doses appeared statistically significantly less effective than methadone in retaining patient in treatment $(\mathrm{RR}=0.82 ; 95 \% \mathrm{CI}$ : 0.69-0.96).

Buprenorphine maintenance versus placebo or methadone maintenance for opioid dependence (Review)

Copyright @ 2008 The Cochrane Collaboration. Published by John Wiley \& Sons, Ltd. 
Low dose buprenorphine is not superior to low dose methadone. High dose buprenorphine does not retain more patients than low dose methadone, but may suppress heroin use better. There was no advantage for high dose buprenorphine over high dose methadone in retention ( $\mathrm{RR}=0.79$; 95\% CI:0.62-1.01), and high dose buprenorphine was inferior in suppression of heroin use. Buprenorphine was statistically significantly superior to placebo medication in retention of patients in treatment at low doses (RR=1.24; 95\% CI: 1.06$1.45)$, high doses ( $R R=1.21$; $95 \% \mathrm{CI}: 1.02-1.44)$, and very high doses ( $\mathrm{RR}=1.52 ; 95 \% \mathrm{CI}: 1.23-1.88)$. However, only high and very high dose buprenorphine suppressed heroin use significantly above placebo.

\section{Authors' conclusions}

Buprenorphine is an effective intervention for use in the maintenance treatment of heroin dependence, but it is not more effective than methadone at adequate dosages.

\section{PLAIN LANGUAGE SUMMARY}

Buprenorphine can reduce heroin use, although it is not as effective as methadone

Methadone is the most widely used replacement for heroin in medically-supported maintenance or detoxification programs. Two other drugs are sometimes used to try and help lower their use of heroin, buprenorphine and LAAM (levo-alpha-acetylmethadol). Buprenorphine is an opioid drug that is not as powerful as heroin and methadone, although the effects may last longer. It is easier to withdraw from buprenorphine than methadone, and can be taken once every two days. The review of trials found that buprehnorphine can reduce heroin use effectively, although it is not as effective as methadone.

\section{B A C K G R O U N D}

Heroin dependence is becoming increasingly prevalent, with associated increases in the spread of infectious disease (e.g., HIV, hepatitis $\mathrm{B}$ and $\mathrm{C}$ ) and overdose deaths. One of the main forms of treatment has been methadone maintenance treatment. As set out by elsewhere (Mattick 1998, Mattick 2003), maintenance treatment with oral methadone appears to be an effective and accepted intervention for opioid (heroin) dependence, and it is widely used in some countries. Yet, methadone maintenance treatment (MMT) has a number of negative characteristics which potentially influence its effectiveness and which have led to an interest in alternative pharmacotherapies and methods of treatment delivery (Mattick 1998). The negative aspects of methadone are set out below.

Methadone is a full opioid agonist at $\mu$-receptors. Thus, one negative aspect of methadone is its potential to produce and/or maintain dependence on opioids, such that patients experience withdrawal if a daily dose is missed, and detoxification can be a lengthy and difficult process which can discourage patients from attempting withdrawal. Additionally, because of its full agonist action, there is no ceiling to the level of respiratory depression or sedation which methadone can induce, and methadone overdose can therefore be fatal. Although it is a long-acting opioid, in some countries and settings, the inconvenience of daily dosing and clinic visits may be unattractive to clients, and restrictions imposed by the daily dosing schedule on clients' general lifestyle and on opportunities to sustain employment may also limit its acceptance to heroin users. The provision of takeaway doses of methadone results in problems of diversion of the drug for illicit use by those not in treatment, although the extent of this problem varies across countries. Finally, heroin users have developed their own "lore" regarding methadone's negative effects, although their views may not always be accurate or favourable. Thus, despite its many advantages, methadone maintenance appears to have limited suitability for some patients. These factors may restrict the ability of methadone to attract certain users into treatment, and the examination of alternative medications to broaden the range of pharmacotherapies been the focus of research in recent years.

There are a number of alternatives to methadone as a maintenance agent in the management of opioid dependence. The most promising of these involve pharmacotherapies which treat patients with a pharmaceutical grade opioid which has a longer duration of action than methadone. These include the opiate partial agonist buprenorphine and the full agonist levo-alpha-acetylmethadol (LAAM) (Mattick 1998). This review focuses on the role of buprenorphine as a maintenance therapy in the management of opioid dependence.

Buprenorphine is a potent synthetic opioid analgesic initially used for the management of acute pain. Pharmacologically, buprenor- 
phine causes morphine-like subjective effects and produces crosstolerance to other opioids. Unlike methadone and heroin (which are full agonists), buprenorphine is a partial agonist and exerts weaker opioid effects at opioid receptor sites. This partial agonist action appears to make buprenorphine safer in overdose. Other benefits of buprenorphine may include an easier withdrawal phase and, because of the longer duration of action, the option of alternate day dosing.

It was during the initial development of buprenorphine as an analgesic in the 1970's that its potential utility as a substitution agent in the treatment of opioid dependence was recognised. Early work (Jasinski 1978) using buprenorphine administered by the subcutaneous route, characterised it as an opioid with low physical dependence liability with a minimal withdrawal syndrome. Subsequently, others (Fudala 1990) provided evidence that buprenorphine does produce a mild to moderate mu-agonist withdrawal syndrome.) It was thought that at doses somewhat greater than those used for analgesia, it could be used in the treatment of opioid dependence (Jasinski 1978).

Evidence on the efficacy of buprenorphine has come from placebocontrolled trials ( Johnson 1995a, Ling 1998), fixed dosing studies of buprenorphine versus methadone maintenance treatment (Bickel 1988; Johnson 1992; Kosten 1993; Ling 1996; Fischer 1999; Uehlinger 1998) and variable dosing studies of buprenorphine versus methadone maintenance treatment (Strain 1994a; Strain 1994b; Mattick 2003). Clinical trials conducted in the U.S. showed buprenorphine to be superior to placebo medication, but when buprenorphine and methadone maintenance were compared in a series of impressive studies using fixed doses of the drugs, the results were mixed. Some of the fixed dose studies showed no difference in efficacy, whereas others showed superiority for methadone and yet others showed the reverse pattern. The investigators in these fixed dose studies frequently concluded that the doses of buprenorphine, or methadone, chosen were too low or that poor induction regimes led to poor retention. A series of variable (or flexible) dose studies have been conducted and shown essentially equivalent results for the two drugs. Given the mixed results, it seems particularly important to attempt a systematic integration of the literature, and separately assessing the fixed and variable dose studies and discussing the results in the light of the differing doses and other individual trial features. Additionally, this review separately summarises the available placebo-controlled trial results.

\section{O B J E C T I VES}

The present systematic review had the objective of providing an evaluation of the buprenorphine maintenance treatment in the management of opioid dependence.

\section{RE S U L T S}

\section{Selection of Studies/Participants/Interventions:}

There were thirteen studies included in this review (2544 participants).

Characteristics of the participants were generally well described. Majority of participants in these studies were male, consistent with the profile of the heroin dependant users generally. They tended to be approximately 30 years of age, again consistent with what is known about heroin users presenting for treatment.

The interventions ranged in duration from 6 weeks through to 52 weeks. By and large the interventions used clinically relevant doses of medication, although as noted earlier a number of the studies used fixed doses of medication and this created some limitations in terms of generalisability to day-to-day clinical practice. Specifically, in day-to-day clinical practice flexible dosing is used.

\section{Quantitative Analysis}

2a. Flexible Dose Buprenorphine versus Flexible Dose Methadone

As noted earlier, the flexible dose studies reported probably provide the best estimate of the likely impact of methadone and buprenorphine in day-to-day clinical practice, as they mirror clinical practice in terms of dose adjustments and in terms of the doses employed in the studies. The six studies included in the flexible dose buprenorphine versus flexible dose methadone analysis (837 participants) showed that methadone was more likely to retain patients than buprenorphine (six studies, 837 participants; $R R=$ 0.82; 95\% CI: 0.69-0.96). The Chi-square test for heterogeneity was not significant. Inspection of the relative risks for retention in treatment for these six flexible dose studies showed two studies had significant poor retention for buprenorphine, but the other four studies showed no statistical significant difference. While there was a difference in retention favouring methadone, turning to the effect of buprenorphine and methadone on drug use, the flexible dose studies showed no significant difference between the two interventions in terms of heroin use, based on results of morphine urinanalysis (six studies, 837 participants; SMD $=-0.12$; 95\% CI: -0.26- 0.02), or in terms of self-reported heroin use (two studies, 326 participants; $\mathrm{SMD}=-0.10 ; 95 \% \mathrm{CI}:-0.32-0.12$ ). Similarly, there was no statistically significant difference between the flexible dose methadone and buprenorphine trials in terms of cocaine positive urines (five studies, 779 participants; SMD $=0.11 ; 95 \%$ CI: $-0.03-0.25$ ) or benzodiazepine positive urines (four studies, 669 participants; $\mathrm{SMD}=0.11 ; 95 \% \mathrm{CI}:-0.04-0.26$ ).

In the one study that reported on criminal activity, there was no statistically difference between the buprenorphine and methadone groups ( $\mathrm{SMD}=-0.14 ; 95 \% \mathrm{CI}:-0.41-0.14)$.

2b. Low Dose Buprenorphine versus Low Dose Methadone

The comparison of low dose buprenorphine and low dose methadone (two studies, 121 participants) indicated no statistically significant difference in retention in treatment $(\mathrm{RR}=0.74 ; 95 \% \mathrm{CI}$ : 
0.52-1.06), nor was there evidence of differences in morphine positive urines and cocaine positive urines based on one trial. There was no difference in self-reported heroin use (one study with 44 participants; $\mathrm{SMD}=-0.28$; 95\% CI: $-0.35-0.90)$.

\section{2c. Low Dose Buprenorphine versus High Dose Methadone}

When low dose buprenorphine is compared to high dose methadone (2 RCTs, 120 participants) there was no statistical difference in retention in treatment $(\mathrm{RR}=0.69$; 95\%CI: 045-1.06). The trials involved did not show heterogeneity. The results show that low dose buprenorphine is not more effective than high dose methadone in retaining patients in treatment, and it is not superior to high dose methadone in suppressing heroin use as indexed by the extent of morphine positive urines (one study, 57 participants; $\mathrm{SMD}=0.88 ; 95 \% \mathrm{CI}: 0.33-1.42$ ). However, the overall effect is only based on one study, as data from the second study (Kosten 1993) concerning the urine results were not available for this review. There was, also, no statistically significant difference of the effect of low dose buprenorphine and high dose methadone beyond the effect of on cocaine, as shown from data on cocaine positive urines (one study, 57 participants; $\mathrm{SMD}=-0.08$; 95\% CI: $-0.60-0.44)$.

There was no statistically significant difference in self-reported heroin use (one study, 38 participants; $\mathrm{SMD}=-0.06$; 95\% CI: $0.70-0.58)$. However, the results from Schottenfeld 1997 on selfreported heroin use, which could not be included in this metaanalysis, did show a significant advantage for high dose methadone (65mg) over low dose buprenorphine (4mg).

\section{2d. High Dose Buprenorphine versus Low Dose Methadone}

When comparing high dose buprenorphine there was one study which favoured high dose buprenorphine in terms of retention, one study that favoured low dose methadone, and two studies showed no statistically significant difference. The test for heterogeneity was significant for the retention data (chi-square $=11.47$, $\mathrm{df}=3, \mathrm{p}=0.0095$ ) therefore no summary measure is provided. However, high dose buprenorphine was superior to low dose methadone in terms of heroin use as shown from morphine positive urines (three studies, 317 participants; $\mathrm{SMD}=-0.23$; 95\%CI: $-0.45-$ 0.01 ), but again the chi-square test for heterogeneity was significant $(\mathrm{p}=0.041)$, even though direction of the estimates were homogeneous. In terms of cocaine positive urines, no benefit was shown for high dose buprenorphine compared with low dose methadone, based on only one study (59 participants).

There was no difference in self-reported heroin use (one study, 37 participants; $\mathrm{SMD}=-0.64 ; 95 \% \mathrm{CI}:-0.06-1.33)$.

\section{2e. High Dose Buprenorphine versus High Dose Methadone}

Comparing high dose buprenorphine and high dose methadone, the data on retention in treatment (5 RCTs, 449 participants) showed no statistical difference between the two interventions
$(\mathrm{RR}=0.79 ; 95 \% \mathrm{CI}: 0.62-1.01)$, but suggest that high doses of buprenorphine are less likely to retain patients than high dose methadone. The trials involved in this comparison (Johnson 1992, Kosten 1993, Ling 1996, Pani 2000, Schottenfeld 1997) did not show any evidence of heterogeneity. High dose buprenorphine was also significantly less able to suppress heroin use as shown by morphine positive urines ( 3 studies, 314 participants: $\mathrm{SMD}=0.27$; 95\%CI: 0.05-0.50) while no statistically significant difference was found in terms of cocaine use based on the cocaine urine analysis results of only one study (57 participants).

There was no difference in self-reported heroin use (two studies, 74 participants; $\mathrm{SMD}=-0.02$; 95\% CI: $-0.48-0.45$ ). This lack of significance is consistent with the results from Schottenfeld 1997 on self-reported heroin use, which could not be included in this meta-analysis, and which did not show a significant advantage for high dose methadone (65mg) over high dose buprenorphine (12mg).

2f. Low dose buprenorphine maintenance versus placebo

Turning to the results on the two trials (487 participants) comparing low dose buprenorphine ( $2 \mathrm{mg}$ or $4 \mathrm{mg}$ ) versus placebo medication (0mg or 1mg, respectively) (Johnson 1995a, Ling 1998), the results showed a benefit for low dose buprenorphine above placebo in terms of retaining patients in treatment $(R R=1.24 ; 95 \%$ CI: 1.06-1.45). However, low dose buprenorphine patients had no less heroin use as indexed by morphine positive urines, cocaine positive urine results, and benzodiazepine positive urines , although these latter two results came from only one of the two trials (Johnson 1995a).

2g. High dose buprenorphine maintenance versus placebo

The results on the two trials (463 participants) comparing high dose $(8 \mathrm{mg})$ buprenorphine versus placebo medication (Johnson 1995a, Ling 1998), the results showed a benefit for buprenorphine above placebo in terms of retaining patients in treatment $(R R=$ 1.21; 95\% CI: 1.02-1.44). Not only were patients better retained by buprenorphine but they had less heroin use as indexed by morphine positive urines. There was an advantage for placebo in terms of cocaine positive urine results, but this is based on only one study (Johnson 1995a). By way of contrast, buprenorphine was superior to placebo in terms of its ability to suppress benzodiazepine use, again this result coming from one trial (Johnson 1995a).

2h. Very high dose buprenorphine maintenance versus placebo

Finally, turning to the one trial (366 participants) comparing very high dose (16mg) buprenorphine versus placebo medication (Ling 1998), the results showed a benefit for buprenorphine above placebo in terms of retaining patients in treatment $(R R=1.52 ; 95 \%$ CI: 1.23-1.88). Not only were the patients in this single trial better retained by buprenorphine, but they had less heroin use when receiving $16 \mathrm{mg}$ of buprenorphine than placebo patients as indexed by morphine positive urines. 
Other measures (e.g. use of other drugs, physical health, and psychological health) were too infrequently and irregularly reported in the literature to be usefully integrated in the quantitative part of this review.

\section{DISCUSSION}

Buprenorphine is superior to placebo in terms of retention based on the large study by Ling 1998 and the study by Johnson 1995a, and it is superior in terms of its ability to suppress heroin use.

When compared with methadone, the results of the meta-analysis indicate that methadone is statistically significantly better able to retain patients than buprenorphine in flexible dosing approaches, the difference being slight in favour of methadone. Methadone is better able to suppress heroin use than buprenorphine, especially if high-dose methadone is used (and vice-versa). Similar conclusions have been reached by other recent meta-analytic reviews of these treatments (West 2000; Barnett 2001).

One explanation which has been advanced by authors in some of the studies included here for the poorer retention in buprenorphine treatment (Fischer 1999, Petitjean 2001) is that they inducted patients too slowly onto buprenorphine and this was the cause of the poorer retention in that medication group. It is possible that retention is affected by too slow induction, and given the apparent relative safety of buprenorphine it may be possible to induct people to higher doses at a more rapid rate and to overcome the problem of slightly poorer retention for buprenorphine compared with methadone. However, there are a number of other possible explanations for the poorer retention on buprenorphine than methadone. In particular, it may well be that buprenorphine, being a partial agonist, does not retain people because it does not have a full opioid effect and is less satisfying to patients. Another possibility is that patients in the initial stages of dosing who have recently ingested heroin suffer a mild withdrawal syndrome by virtue of buprenorphine (a partial agonist) displacing heroin (a full agonist) from opioid receptors in the central nervous system, and this mild withdrawal may lead patients to leave treatment. A further possibility is that buprenorphine is simply easier to withdraw from and, on that basis, patients are more at liberty to leave treatment without the severe withdrawal syndrome that can accompany methadone withdrawal. Of course, these factors may all act together to cause buprenorphine to have a slightly poorer outcome in terms retention than methadone. Future research should be undertaken to address this particular issue.

These results show that, despite the effectiveness of buprenorphine when compared with placebo, when methadone is given in high doses buprenorphine is not a better treatment. These findings could be taken to suggest that buprenorphine will be inferior to methadone in day-to-day practice, but we point out that some of doses used in the high dose methadone conditions are rarely used in day-to-day clinical practice. If it were possible to get clinicians and patients to agree to prescribe and take high dose methadone then the clinical implication of this may be important, but the reality is that most often methadone is prescribed in the range of an average of 50 to 60 milligrams. At this level, as shown in the flexible dose studies earlier, there does not appear to be any reliable difference in heroin use between methadone and buprenorphine, overall.

The clinical trials represented in this review are of reasonable quality, and whilst many of them did not fully explain how randomisation was concealed, they appear to have used doses which are clinically relevant and to have treated patients for significant periods of time. Based on the nature of the trials, it would appear the external validity or generalisability of the results is quite good, particularly from those trials which have used large sample sizes and adequate doses.

\section{AUTHORS'CONCLUSIONS}

\section{Implications for practice}

The implication of the results of the meta-analytic review conducted and reported herein are clear for clinical practice. Buprenorphine is an effective treatment for heroin use in a maintenance therapy approach compared with placebo. However, methadone maintenance treatment at high dose is associated with higher rates of retention in treatment and better suppression of heroin than buprenorphine maintenance treatment. Buprenorphine maintenance should be supported as a maintenance treatment, only where higher doses of methadone cannot be administered. The reasons for not applying the best available treatment should be investigated rather than promoting less effective treatment approaches.

Given buprenorphine's different pharmacological properties, it may have advantages in some settings and under some policies where its relative safety and alternate-day administration are useful clinically compared to methadone.

\section{Implications for research}

Overall, the quality of the studies included in this meta-analytic review was quite good. The trials did use sample sizes which varied from relatively small to quite large, but they were mostly doubleblind trials. There does not appear to be any need for further randomised control trials of the relative efficacy of methadone compared with buprenorphine. There does appear to be a need to undertake studies which will clarify retention in the first few weeks or months of treatment in buprenorphine versus methadone. One way of addressing this issue would be to compare a standard induction as used in some of the trials reported herein with a rapid induction onto buprenorphine, with the potential to have a further comparison of induction onto methadone. Problems in the 
methods of induction onto buprenorphine within the trials analysed might partly explain the inferiority of buprenorphine shown in this review. It would be ideal if such a trial were to be conducted under double blind conditions, particularly in terms of the rapid versus standard induction onto buprenorphine. Other outcome measures such as self-reported drug use, criminal activity, physical health, and psychological health which were too infrequently and irregularly reported in the literature to be analysed in the current review could be included in future studies.

\section{ACKNOW LEDGEMENTS}

We acknowledge the contribution of the investigators from the trials who transformed the urine data to allow it to be incorporated into this review. We also acknowledge Marica Ferri, the Coordinator of the Drugs and Alcohol group for her attention to the process of the review and contribution to the work.

\section{R E F E R E N C E S}

\section{References to studies included in this review}

Fischer 1999 \{published and unpublished data\} Fischer G, Gombas W, Eder H, Jagsch R, Peternell A, Stuehlinger G, Pezawas L, Aschauer H, Kasper S. Buprenorphine versus methadone maintenance for the treatment of opioid dependence. Addiction 1999;94: $1337-1347$

Johnson 1992 \{published and unpublished data\} Johnson R, Jaffe J, Fudala P. A controlled trial of buprenorphine treatment for opioid dependence. JAMA 1992;267:2750-2755. [MEDLINE: 1992251936].

Johnson 1995a \{published and unpublished data\} Johnson RE, Eissenberg T, Stitzer M, Strain E, Liebson I, Bigelow G. A placebo controlled trial of buprenorphine as a treatment for opioid dependence. Drug and Alcohol Dependence 1995;40:17-25.

Johnson 2000 \{published and unpublished data\} Johnson RE, Chutuape MA, Strain, EC, Walsh SL, Stitzer ML, Begelow GE. First Randomized Controlled Trial (RCT) of Methadone (M), Levomethadyl Acetate (LAAM) and Buprenorphine (BUP) in Opioid Dependence Treatment. Clinical Pharmacology \& Therapuetics 1999;65 (2):145-.

Kosten 1993 \{published data only\} Kosten T, Schottenfeld R, Ziedonis D, Falcioni J. Buprenorphine versus methadone maintenance for opioid dependence. J Nerv Ment Dis 1993;181:358-364. [MEDLINE: 1993274288].

Ling 1996 \{published and unpublished data\} Ling W, Wesson D, Charuvastra C, Klett J. A controlled trial comparing buprenorphine and methadone maintenance in opioid dependence. Arch Gen Psychiatry 1996;53:401-407. [MEDLINE: 1996212784].

Ling 1998 \{published and unpublished data\} Ling W, Charuvastra C, Collins J, Batki S, Brown L, Kintaudi P, Wesson D, McNicholas L, Tusel D, Malkerneker U, Renner J, Santos E, Casadonte P, Fye C, Stine S, Wang $\mathrm{R}$, Segal D. Buprenorphine maintenance treatment of opiate dependence: a multicenter, randomized clinical trial. Addiction 1998;93:475-486.
Mattick 2003 \{published data only\}

* Mattick RP, Ali R, White J, O'Brien S, Wolk S, Danz, C. Buprenorphine versus methadone maintenance therapy: a randomised double-blind with 405 opioid-dependent patients. Addiction 2003;98:441-452.

Pani 2000 \{published data only\} Pani P, Maremmani I, Pirastu R, Tagliamonte A, Gessa G. Buprenorphine: a controlled trial in the treatment of opioid dependence. Drug and Alcohol Dependence 2000;60:39-50.

Petitjean 2001 \{published and unpublished data\} Petitjean S, Stohler R, Deglon J, Livoti S, Waldvogel D, Uehlinger C, Ladewig D. Double blind randomized trial of buprenorphine and methadone in opiate dependence. Drug and Alcohol Dependence 2001;62:97-104.

Schottenfeld 1997 \{published and unpublished data\} Schottenfeld R, Pakes J, Oliveto A, Ziedonis D, Kosten T. Buprenorphine vs methadone maintenance treatment for concurrent opioid dependence and cocaine abuse. Arch Gen Psychiatry 1997;54(8):713-20. [MEDLINE: 1997429160].

Strain 1994a \{published and unpublished data\} Strain E, Stitzer M, Liebson I, Bigelow G. Comparison of buprenorphine and methadone in the treatment of opioid dependence. Am J Psychiatry 1994;151(7):1025-1030. [MEDLINE: 1994279720].

Strain 1994b \{published and unpublished data\} Strain E, Stitzer M, Liebson I, Bigelow G. Buprenorphine versus methadone in the treatment of opioid dependent cocaine users. Psychopharmocology 1994;116(4):401-6. [MEDLINE: 1995215552].

\section{References to studies excluded from this review}

\section{Bickel 1988}

Bickel W, Stitzer M, Bigelow G, Liebson I, Jasinski D, Johnson R. A clinical trial of buprenorphine: Comparison with methadone in the detoxification of heroin addicts. Clin Pharmacol Ther 1988;43(1):72-78. [MEDLINE: 1988081296].

\section{Bouchez 1998}

Bouchez J, Beauverie P, Touzeau D. Substiitution with buprenorphine in methadone- and morphine sulfate 
dependent patients. European Addiction Research 1998;4

(suppl1):8-12.

Eder 1998

Eder H, Fischer G, Gombas W, Jagsch R, Stuehlinger G, Kasper S. Comparison of buprenorphine and methadone maintenance in opiate addicts. Eur Addict Res 1998;4(1): 3-7.

\section{Johnson 1995b}

Johnson RE, Eissenberg T, Stitzer ML, Strain EC, Liebson IA, Bigelow GE. Buprenorphine treatment of opioid dependence: Clinical trial of daily versus alternate-day dosing. Drug and Alcohol Dependence 1995;40:27-35.

O’Connor 1998

O'Connor P, Oliveto A, Shi J, Triffleman E, Carroll K, Rounsaville B, Pakes J, Schottenfeld R. A randomized trial of buprenorphine maintenance for heroin dependence in a primary care clinic for substance users versus a methadone clinic. Am J Medicine 1998;105(2):100-5.

Oliveto 1994

Oliveto A, Kosten T, Schottenfeld R, Ziedonis D, Falcioni $\mathrm{J}$. Cocaine use in buprenorphine vs methadone maintained patients. Am J Addictions 1994;3(1):43-48.

\section{Schottenfeld 1998}

Schottenfeld R, Pakes J, Kosten T. Prognostic factors in Buprenorphine versus Methadone maintained patients. J Nerv Ment Dis 1998;186(1):35-43. [MEDLINE: 1998118328].

\section{Stine 1994}

Stine M, Kosten T. Reduction of opiate withdrawal like symptoms by cocaine abuse during methadone and buprenorphine maintenance. Am J Drug Alcohol Abuse 1994;20(4):445-58. [MEDLINE: 1995133568].

\section{Strain 1996}

Strain E, Stitzer M, Liebson I, Bigelow G. Buprenorphine versus methadone in the treatment of opioid dependence: self reports, urinalysis, and addiction severity index. $J$ Clin Psychopharmacology 1996;16(1):58-67. [MEDLINE: 1996431336].

\section{Uehlinger 1998}

Uehlinger C, Deglon J, Livoti S, Petitjean S, Waldvogel D, Ladewig D. Comparison of buprenorphine and methadone in the treatment of opioid dependence. Eur Addict Res 1998;4(1):13-18.

\section{Additional references}

\section{Barnett 2001}

Barnett P, Rodgers J, Bloch D. A meta-analysis comparing buprenorphine to methadone for treatment of opiate dependence. Addiction 2001;96:683-690.

\section{Fudala 1990}

Fudala PJ, Jaffe JH, Dax EM, Johnson RE. Use of buprenorphine in the treatment of opioid addiction. II. Physiologic and behavioral effects of daily and alternateday administration and abrupt withdrawal. Clin Pharmacol Ther 1990;47:525-34.

\section{Jasinski 1978}

Jasinski D, Pevnick J, Griffith J. Human pharmacology and abuse potential of the analgesic buprenorphine. Arch Gen Psychiatry 1978;35:501-506.

\section{Mattick 1998}

Mattick RP, Oliphant D, Ward J, Hall W. The effectiveness of other opioid replacement therapies: LAAM, heroin, buprenorphine, naltrexone and injectable maintenance. Methadone maintenance and other opioid replacement therapies. Amsterdam: Harwood Academic Publishers, 1998:123-160.

West 2000

West S, O’Neal K, Graham C. A meta-analysis comparing the effectiveness of buprenorphine and methadone. Journal of Substance Abuse Treatment 2000;12:405-414.

* Indicates the major publication for the study

\section{SOURCES OF SUPPORT}

\section{External sources of support}

- Commonwealth Department of Health and Aged Care, Canberra AUSTRALIA 


\section{Internal sources of support}

- National Drug and Alcohol Research Centre, University of New South Wales, Sydney AUSTRALIA

\section{NDEX TERMS}

\section{Medical Subject Headings (MeSH)}

Buprenorphine [*therapeutic use]; Methadone [ ${ }^{*}$ therapeutic use]; Narcotic Antagonists [*therapeutic use]; Narcotics [*therapeutic use]; Opioid-Related Disorders [* rehabilitation]; Randomized Controlled Trials

\section{MeSH check words}

Humans 